\title{
Inferring Within-Host Bottleneck Size: A Bayesian Approach
}

\author{
R. Dybowski ${ }^{1, *}$, O. Restif ${ }^{1}$, D.J. Price ${ }^{1}$, P. Mastroeni ${ }^{1}$ \\ ${ }^{1}$ Department of Veterinary Medicine, University of Cambridge, \\ Madingley Road, Cambridge, CB3 0ES, UK \\ *Corresponding author: rd460@cam.ac.uk
}

\begin{abstract}
A number of approaches exist for bottleneck-size estimation with respect to within-host bacterial infections; however, some are more appropriate than others under certain circumstances. A Bayesian comparison of several approaches is made in terms of the availability of isogenic multitype bacteria (e.g., WITS), knowledge of post-bottleneck dynamics, and the suitability of dilution with monotype bacteria. The results are summarised by a guiding flowchart.

A sampling approach to bottleneck-size estimation is also introduced.
\end{abstract}

\section{Keywords}

Bottlenecks

Bayesian inference

Wildtype isogenic tagged strains (WITS)

Salmonella

\section{Introduction}

The outcome of an infection processes is usually underlain by a fine balance between the virulence mechanisms of the pathogen and the resistance of the 
host. The presence of physical, immunological or therapeutic barriers poses constraints to the ability of bacteria to divide and disseminate within the host organism. Suppose that we have a population $\mathcal{P}_{0}$ of bacteria. When a subset of $\mathcal{P}_{0}$ is inactivated by an antibiotic or an immune response, or subject to an anatomical barrier to transmission, this can result in a substantially smaller population $\mathcal{P}_{1}$ (commonly known as a bottleneck) and, after the occurrence of the bottleneck, the bacteria of $\mathcal{P}_{1}$ can grow to form a new population $\mathcal{P}_{2}$.

Understanding the site, nature and size of bottlenecks in infectious disease processes is important to rationally design prevention strategies and treatments to control the spread of the infection within a given host. In fact, classes of vaccines and therapeutic compounds differ significantly in how they restrain an infection process with respect to the control, for example, of microbial killing or division rates and the spread within and between organs.

Bottlenecks have been the focus of a number of articles, and Abel et al. (2015) provide a biologically motivated introduction to bottlenecks. Specific experimental studies that have shown bottlenecks using isogenic tagged strains include Grant et al. (2008) in the early stage of salmonellosis in mice, Schwartz et al. (2011) in urinary tract Escherichia coli infection in mice, Lowe et al. (2013) during Bacillus anthracis colonisation in mice, Kaiser et al. (2014) with Salmonella Typhimurium crossing the intestinal barrier in mice, Lim et al. (2014) also with Salmonella, Gerlini et al. (2014) and Kono et al. (2016) with invasive Streptococcus pneumoniae, and Abel et al. (2015) with Vibrio cholerae in the intestinal tract. However, in spite of the number of studies that have been made involving bottlenecks, there has not been a unified study of the various analytical methods used for tagged/multitype experimental studies, which is the motivation of this study.

\section{Monotype populations}

\subsection{Posterior bottleneck distributions}

Of interest is estimating the size of a bottleneck given observations made after the bottleneck, and possibly also before it, in a Bayesian framework. This can be expressed as the posterior probabilities

$$
p \text { (bottleneck size|observation after bottleneck) }
$$


and

$$
\begin{array}{r}
p(\text { bottleneck size } \mid \text { observation before bottleneck \& } \\
\text { observation after bottleneck) . }
\end{array}
$$

The benefit of taking the Bayesian approach of using posterior probability distributions is that such distributions not only give estimates for the most probable bottleneck sizes (in terms of the modes of the distribution) but they also express the uncertainty through the variance of the distributions.

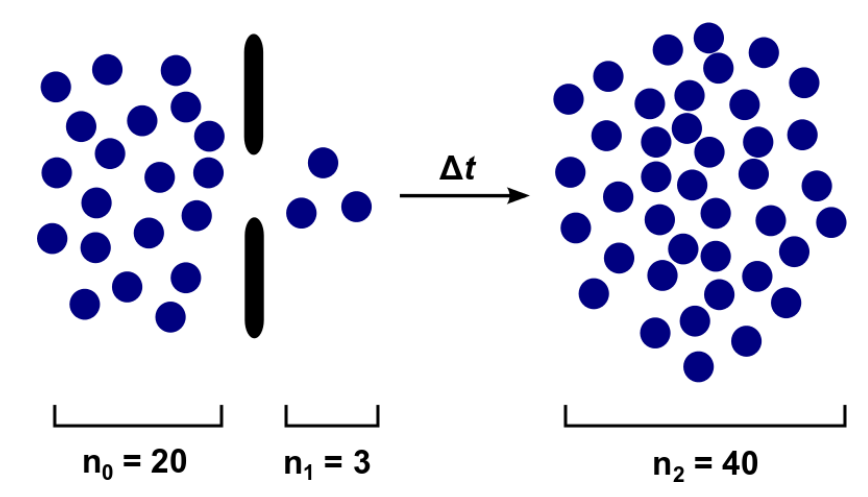

Figure 1: Diagrammatic representation of a bottleneck. The bottleneck is derived from a pre-bottleneck population by random sampling without replacement. A post-bottleneck population arises after time $\Delta t$ according to a defined growth mechanism. $n_{1}$ is the size of the bottleneck, $n_{0}$ the size of the pre-bottleneck population, and $n_{2}$ the size of the post-bottleneck population.

Abel et al. (2015) estimated bottleneck size with respect to multitype populations by equating it to the effective population size as estimated by Krimbas and Tsakas (1971), which uses the standardised covariance of allele frequency due to a bottleneck. Although Abel et al. found this approach successful in the context of the within-host dynamics of Vibro cholera, both Pamilo and Varvio-Aho (1980) and Sourdis and Krimbas (1980) caution that bottleneck-size estimation by the Krimbas-Tsakas method can be unreliable unless sample size is sufficiently large.

Let $n_{1}$ be the size of a bottleneck $\mathcal{P}_{1}$ and $n_{2}$ the size of a post-bottleneck population $\mathcal{P}_{2}$ after it (Figure 1). For the posterior distribution $p\left(n_{1} \mid n_{2}\right)$, 
Bayes' theorem gives

$$
p\left(n_{1} \mid n_{2}\right) \propto p\left(n_{1}\right) p\left(n_{2} \mid n_{1}\right)
$$

and if we assume that the priors $p\left(n_{1}\right)$ are equally likely we then have the expression

$$
p\left(n_{1} \mid n_{2}\right)=\frac{p\left(n_{2} \mid n_{1}\right)}{\sum_{n_{1}} p\left(n_{2} \mid n_{1}\right)} .
$$

Consider $p\left(n_{2} \mid n_{1}\right)$ for (3). Suppose that we can simulate the occurrence of $n_{2}$ resulting stochastically from $n_{1}$ after time interval $\Delta t$ (Figure 5 ) according to a set of parameters $\boldsymbol{\theta}$ for the dynamics; for example, in the context of the birth-death-migration process shown in Figure 2 (Grant et al., 2008; Kaiser et al., 2014; Coward et al., 2014; Dybowski et al., 2015). Such a simulation can be achieved by a Gillespie stochastic simulation algorithm (Gillespie, 1997). If we obtain a finite number of such simulations,

$$
\left(n_{2}\right)_{1},\left(n_{2}\right)_{2}, \ldots,\left(n_{2}\right)_{m} \stackrel{i . i . d}{\sim} \text { Gillespie }\left(n_{1}, \Delta t, \boldsymbol{\theta}\right)
$$

our task is then to estimate the probability mass function $p\left(n_{2} \mid n_{1}, \boldsymbol{\theta}\right)$ from $\left\{\left(n_{2}\right)_{1},\left(n_{2}\right)_{2}, \ldots,\left(n_{2}\right)_{m}\right\}$, which can then be used for $(3)$.

Estimating $p\left(n_{2} \mid \cdot, \cdot\right)$ from the sparse sample $\left\{\left(n_{2}\right)_{1},\left(n_{2}\right)_{2}, \ldots,\left(n_{2}\right)_{m}\right\}$ can be attempted using local polynomial smoothing (Simonoff, 1996). A recent development in this area is the local polynomial smoothing proposed by Jacob and Oliveira (2011), which is effective for small sample sizes.

Let $\omega=\left\{\left(n_{2}\right)_{1},\left(n_{2}\right)_{2}, \ldots,\left(n_{2}\right)_{m}\right\}$, and let the values of $\omega$ be placed in $k$ successive cells $C_{1}, \ldots, C_{k}$, with all occurrences of $\min (\omega)$ being placed in cell $C_{1}$, all occurrences of $\min (\omega)+1$ in cell $C_{2}, \ldots$, and all occurrences of $\max (\omega)$ in $C_{k}$. The aim is to estimate the true cell probability $\pi_{l}$ for each cell $C_{l}$ based on the finite observations $\omega$. A straightforward estimator of $\pi_{l}$ is, of course, the relative frequency $N_{l} / m$, where $N_{l}$ is the number of values occupying cell $C_{l}$; however, using local polynomials of degree $d$, a more accurate estimation is provided by Jacob and Oliveira (2011):

$$
\widehat{\pi}_{l}(d)=\frac{1}{k h} \sum_{j=1}^{k} L_{l, d}\left(\frac{x_{j}-x_{l}}{h}\right) \frac{N_{j}}{m},
$$

where $L_{l, d}(\cdot)$ is the local $d$-degree polynomial estimator for the probability of cell $C_{l}$, and $x_{j}=(j-1 / 2) / k$ for $j=1, \ldots, k$. Based on the work by Ruppert 
and Wand (1994) on locally weighted regression, Aerts et al. (1997a, 1997b) express $L_{l, d}(\cdot)$ by

$$
L_{l, d}(u)=\frac{\left|M_{l, d}(u)\right|}{\left|N_{l, d}\right|} K(u)
$$

with $N_{l, d}$ the $(d+1) \times(d+1)$-matrix having the $(r, s)$ entry given by

$$
\left(N_{l, d}\right)_{r, s}=\frac{1}{k h} \sum_{i=1}^{k}\left(\frac{x_{i}-x_{l}}{h}\right)^{r+s-2} K\left(\frac{x_{i}-x_{l}}{h}\right) .
$$

Matrix $M_{l, d}(u)$ is the same as $N_{l, d}$ but with the first column replaced by $\left(1, u, \ldots, u^{d}\right)^{T}$. The width of density function $K(\cdot)$ is controlled by $h$.

Both this technique for local polynomial smoothing and Gillespie simulation were used in Algorithm 1 for the estimation of $p\left(n_{1} \mid n_{2}\right)$.

Algorithm 1 Estimation of $p\left(n_{1} \mid n_{2}\right)$.

Input: Post-bottleneck population size $n_{2}$, and Gillespie simulation parameters $\Delta t$ and $\boldsymbol{\theta}$.

Output: An estimate of $p\left(n_{1} \mid n_{2}\right)$ for $n_{1}=1, \ldots, n_{\max }$.

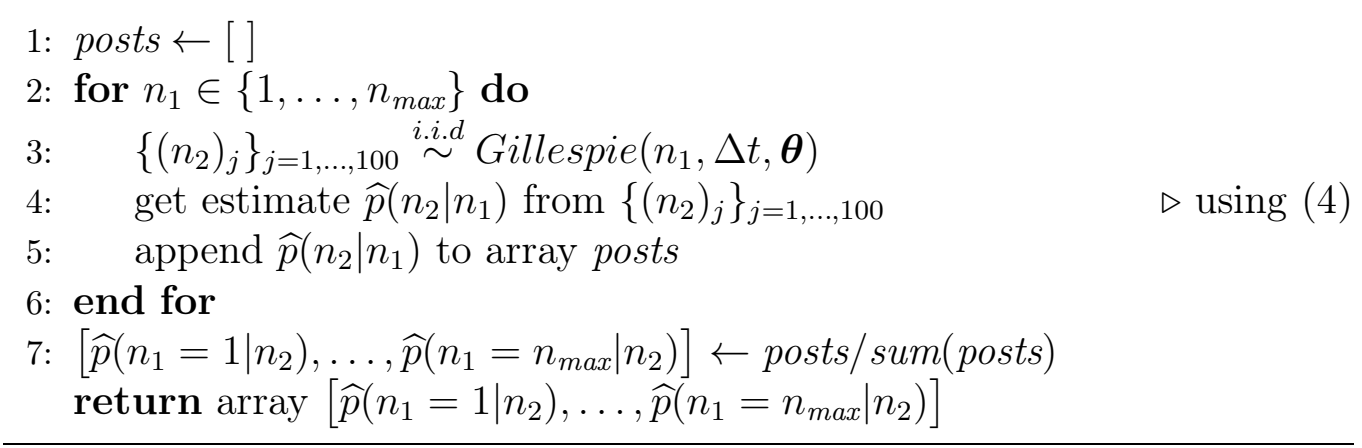




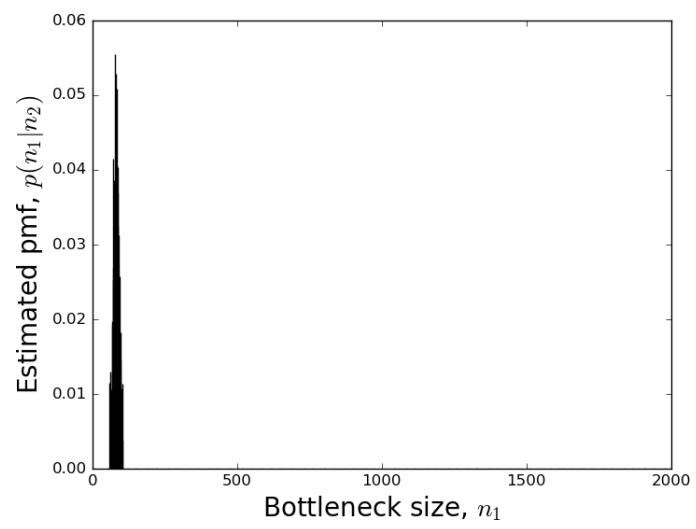

(a)

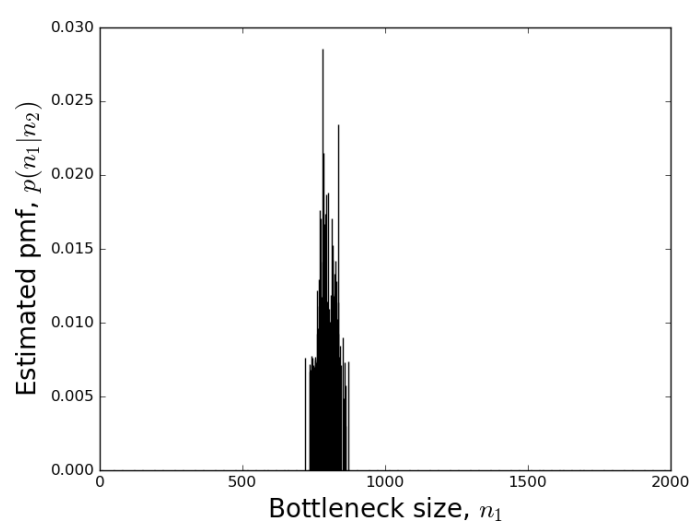

(b)

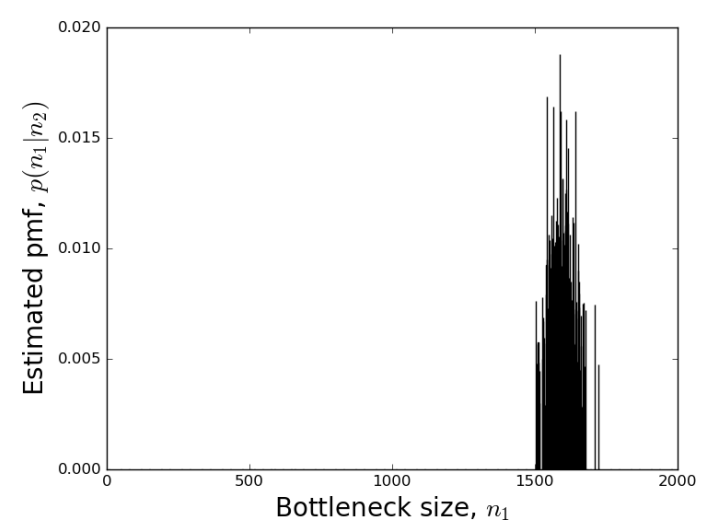

(c)

Figure 2: Posterior bottleneck-size distributions $p\left(n_{1} \mid n_{2}\right)$ estimated using Algorithm 1. Bottlenecks of size (a) $n_{1}=80$, (b) $n_{1}=800$, and (c) $n_{1}=1600$, were artificially induced. 


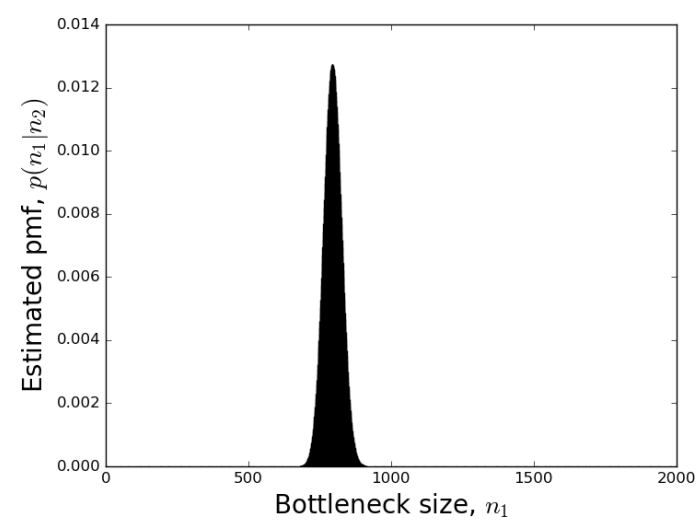

Figure 3: Posterior bottleneck-size distribution estimated using convolution of eight posterior distributions associated with the eight WITS. Target $n_{1}=800$. Summary statistics are mode 793, mean 793.8, median 795 and variance 980.1. See Section 3 for details.

\subsection{Example}

Salmonella enterica is the main cause of salmonellosis, and its pathogenesis is under extensive research (Dougan \& Baker, 2014). When S. enterica enters the bloodstream of a host, bacteria spread to a number of organs including the liver and the spleen (Figure 4). In researching this process using a mouse model, Grant et al. (2008) estimated the division, death, clearance and immigration rates of the bacterium at different times. 


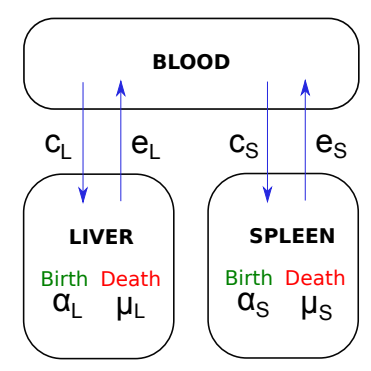

Figure 4: Schematic representation of the spread of $S$. enterica from the blood and between the liver and the spleen. The dynamics is governed by a set of parameters $\boldsymbol{\theta}$; namely, the per capita division rates $\left(\alpha_{L}, \alpha_{S}\right)$, death rates $\left(\mu_{L}, \mu_{S}\right)$, immigration rates $\left(c_{L}, c_{S}\right)$ and clearance rates $\left(e_{L}, e_{S}\right)$.

To demonstrate the efficacy of Algorithm 1 to detect bottlenecks of size $n_{1}^{\star}=1,2,4,8,80,800$ and 1600 (caused by a hypothetical antibiotic treatment) in an organ such as the liver of a mouse, a value $n_{2}^{\star}$ for $n_{2}$ was first derived from $n_{1}^{\star}$ and then the posterior distribution $p\left(n_{1} \mid n_{2}^{\star}\right)$ was estimated from $n_{2}^{\star}$ using Algorithm 1 as follows:

1: Choose a target bottleneck size $n_{1}^{\star} \in\{1,2,4,8,80,800,1600\}$

2: $\left\{\left(n_{2}\right)_{j}\right\}_{j=1, \ldots, 101} \stackrel{\text { i.i.d }}{\sim}$ Gillespie $\left(n_{1}^{\star}, \Delta t, \boldsymbol{\theta}\right) \triangleright 101 n_{2}$ values derived from $n_{1}^{\star}$

3: Set $n_{2}^{\star}$ to the median of $\left\{\left(n_{2}\right)_{j}\right\}_{j=1, \ldots, 101}$

4: Obtain $\widehat{p}\left(n_{1} \mid n_{2}^{\star}\right)$ using Algorithm 1

5: Compare $\widehat{p}\left(n_{1} \mid n_{2}^{\star}\right)$ with target $n_{1}^{\star}$

The 101 Gillespie simulations were conducted using an inoculum of 1000 bacteria at $t=0$ (equivalent to using 101 mice). An infection was allowed to progress according to $\boldsymbol{\theta}$ using the parameter values published by Grant et al. (2008) (i.e., per capita division, death, emigration and immigration rates), but at $t=24$ hours, the number of bacteria in the liver was changed to $n_{1}$ within the Gillespie simulation. The growth period $\Delta t$ was set to 12 hours. Estimates of $p\left(n_{2} \mid n_{1}, \boldsymbol{\theta}\right)$ for (3) were provided by (4) using local polynomial smoothing with an Epanechnikov kernel density function and local polynomials of degree $d=1$.

Figure 2 presents the resulting estimated posterior probabilities, and Table 1 shows that the medians of the posteriors agreed within $1 \%$ of the true 
bottleneck sizes.

Table 1: The accuracy of Algorithm 1 in terms of summary statistics for the estimated posterior distribution $\widehat{p}\left(n_{1} \mid n_{2}^{\star}\right)$. See Section 2.2 for details.

\begin{tabular}{|c|c|c|c|c|c|}
\hline Target $n_{1}^{\star}$ & $n_{2}^{\star}$ & Mode & Mean & Median & SD \\
\hline 1 & 3 & 1 & 1.9 & 1 & 1.1 \\
2 & 8 & 2 & 2.7 & 2 & 1.4 \\
4 & 17 & 4 & 4.5 & 4 & 1.9 \\
8 & 35 & 7 & 8.4 & 8 & 3.2 \\
80 & 376 & 76 & 79.2 & 81 & 9.5 \\
800 & 3731 & 782 & 794.9 & 794 & 30.8 \\
1600 & 7507 & 1587 & 1595.1 & 1597 & 42.3 \\
\hline
\end{tabular}

\subsection{Inclusion of a pre-bottleneck population}

What if the size $n_{0}$ of the pre-bottleneck population $\mathcal{P}_{0}$ is also available to us, or is assumed? The posterior distribution for $n_{1}$ becomes

$$
\begin{aligned}
p\left(n_{1} \mid n_{0}, n_{2}\right) & \propto p\left(n_{1} \mid n_{0}\right) p\left(n_{2} \mid n_{0}, n_{1}\right) \\
& =p\left(n_{1} \mid n_{0}\right) p\left(n_{2} \mid n_{1}\right)
\end{aligned}
$$

Bacteria are either killed by an antibiotic or not; therefore, with regard to $p\left(n_{1} \mid n_{0}\right)$, a simple assumption is that this probability is given by the binomial probability distribution

$$
p\left(n_{1} \mid n_{0} ; \pi\right)=\left(\begin{array}{c}
n_{0} \\
n_{1}
\end{array}\right) \pi^{n_{1}}(1-\pi)^{n_{0}-n_{1}}
$$

where $\pi$ is the probability that a bacterium will be included in the bottleneck; however, $\pi$ is not known a priori. Furthermore, (5) implies that the expected size $n_{1}$ of the bottleneck is a linear function of $n_{0}$ for all $n_{0}$,

$$
\mathbb{E}\left[n_{1} \mid n_{o} ; \pi\right]=n_{0} \pi
$$

but Abel et al. (2015) warn that alternative scenarios could exist. 


\section{Multitype populations}

A multitype population of bacteria is possible by using phenotypically identical bacterial strains where each strain carries a different DNA signature tag in the same noncoding region of the chromosome (Crimmins \& Isberg, 2012). An example of this is the use of wild-type isogenic tagged strains (WITS) (Grant et al., 2008) which we will consider herein.

Suppose now that bacterial pre-bottleneck population $\mathcal{P}_{0}$ is composed of eight WITS: $w_{0}^{[1]}, \ldots, w_{0}^{[8]}$, where $w_{0}^{[i]} \geq 0$ is the number of bacteria tagged with the $i$-th WITS tag. The population is reduced to bottleneck $\mathcal{P}_{1}$ with WITS distribution $w_{1}^{[1]}, \ldots, w_{1}^{[8]}$, and population $\mathcal{P}_{2}$ resulting from the growth of $\mathcal{P}_{1}$ has WITS distribution $w_{2}^{[1]}, \ldots, w_{2}^{[8]}$. As shown in Figure 5 , the distribution of WITS in $\mathcal{P}_{2}$ can be very different to that present in $\mathcal{P}_{0}$ because of the stochastic variation of $\mathcal{P}_{1}$ (Abel et al., 2015), and possibly also from $\mathcal{P}_{1}$ to $\mathcal{P}_{2}$.

Given the WITS distribution $w_{2}^{[1]}, \ldots, w_{2}^{[8]}$ of a post-bottleneck population, estimated posterior distributions $\widehat{p}\left(w_{1}^{[1]} \mid w_{2}^{[1]}\right), \ldots, \widehat{p}\left(w_{1}^{[8]} \mid w_{2}^{[8]}\right)$ can be obtained for each of the WITS independently of each other using Algorithm 1, with $w_{1}^{[j]}$ being used in the algorithm place of $n_{1}$, and $w_{2}^{[j]}$ in place of $n_{2}$.

The total size $n_{1}$ of a bottleneck composed of WITS is given by the sum $w_{1}^{[1]}+\cdots+w_{1}^{[8]}$. There are two approaches to estimating $n_{1}$ : (a) use the sum $w_{2}^{[1]}+\cdots+w_{2}^{[8]}$ for $n_{2}$, ignore the WITS tags and estimate $p\left(n_{1} \mid n_{2}\right)$ using Algorithm 1; (b) determine the posterior mass function for the sum $n_{1}=w_{1}^{[1]}+\cdots+w_{1}^{[8]}$ by applying convolution successively to the individual WITS posterior distributions $\widehat{p}\left(w_{1}^{[1]} \mid w_{2}^{[1]}\right), \ldots, \widehat{p}\left(w_{1}^{[8]} \mid w_{2}^{[8]}\right)$.

If $X_{1}$ and $X_{2}$ are two independent integer-valued random variables with distribution functions $p_{1}=p\left(X_{1}\right)$ and $p_{2}=p\left(X_{2}\right)$, and $Z=X_{1}+X_{2}$, the distribution function $p(Z)$ is given by

$$
p(Z=z)=\left(p_{1} \otimes p_{2}\right)(z)=\sum_{x} p\left(X_{1}=x\right) p\left(X_{2}=z-x\right),
$$

where $\otimes$ is the convolution operator.

Because the convolution operator is commutative, we can extend its use to sums of more than two random variables, $Z=X_{1}+X_{2}+\cdots+X_{m}$, by repeatedly applying the operator:

$$
\begin{aligned}
p(Z=z) & =\left(p_{1} \otimes p_{2} \otimes \cdots \otimes p_{m}\right)(z) \\
& =\left(\left(\cdots\left(p_{1} \otimes p_{2}\right)(z) \otimes \cdots\right) \otimes p_{m}\right)(z)
\end{aligned}
$$


To examine the effect of using convolution, we used target value $n_{1}^{\star}=$ 800 with $w_{1}^{[1] \star}=10, \ldots, w_{1}^{[8] \star}=10$. Convolution was applied to the estimated posteriors $\widehat{p}\left(w_{1}^{[1]} \mid w_{2}^{[1] \star}\right), \ldots, \widehat{p}\left(w_{1}^{[8]} \mid w_{2}^{[8] \star}\right)$ via Fourier transformation, but the resulting estimated posterior $\widehat{p}\left(n_{1} \mid w_{2}^{[1] \star}, \ldots, w_{2}^{[8] \star}\right)=\widehat{p}\left(w_{1}^{[1]}+\cdots+\right.$ $\left.w_{1}^{[8]} \mid w_{2}^{[1] \star}, \ldots, w_{2}^{[8] \star}\right)$ was no better than the posterior $\widehat{p}\left(n_{1} \mid n_{2}\right)$ obtained by ignoring the WITS other than being smoother (Fig 6).

\subsection{Inclusion of a multitype pre-bottleneck population}

Suppose that we know, or are able to assume, the composition $\mathbf{w}_{0}$ of a prebottleneck multitype population as well as that of a post-bottleneck population. In this case, the posterior of the bottleneck size is $p\left(n_{1} \mid \mathbf{w}_{0}, \mathbf{w}_{2}\right)$.

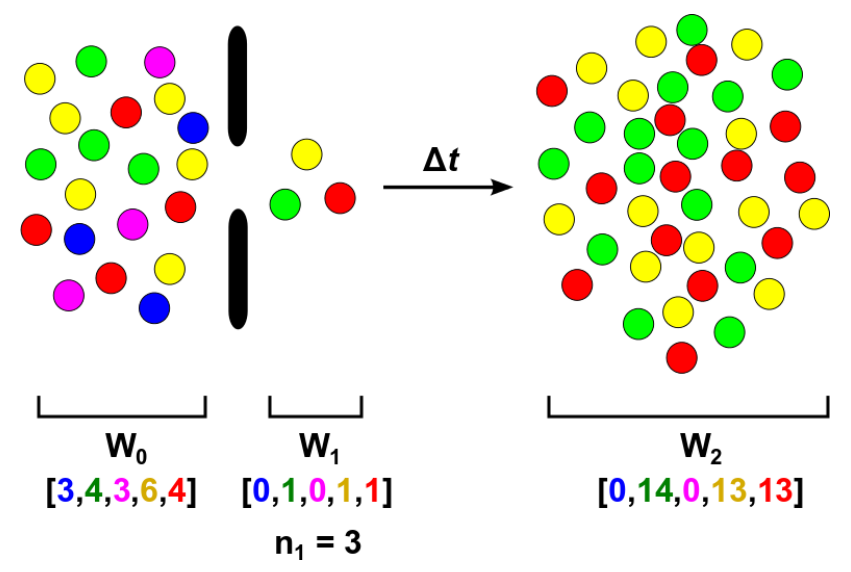

Figure 5: Diagrammatic representation of a bottleneck and its propensity to stochastic variability in terms of both its size $\left(n_{1}\right)$ and the composition of its population $\left(\mathbf{w}_{1}\right)$. The array of numbers below each population states the number of tagged bacteria present in that population. 
Now,

$$
\begin{aligned}
p\left(n_{1} \mid \mathbf{w}_{0}, \mathbf{w}_{2}\right) & =p\left(\bigvee_{\substack{\mathbf{w}_{1} \\
\text { s.t. } \operatorname{sum}\left(\mathbf{w}_{1}\right)=n_{1}}} \mathbf{w}_{1} \mid \mathbf{w}_{0}, \mathbf{w}_{2}\right) \\
& =\sum_{\substack{\mathbf{w}_{1} \\
\text { s.t. } \operatorname{sum}\left(\mathbf{w}_{1}\right)=n_{1}}} p\left(\mathbf{w}_{1} \mid \mathbf{w}_{0}, \mathbf{w}_{2}\right),
\end{aligned}
$$

where $\operatorname{sum}\left(\mathbf{w}_{1}\right)=\sum_{i} w_{1}^{[i]}$. From Bayes' theorem,

$$
\begin{aligned}
p\left(\mathbf{w}_{1} \mid \mathbf{w}_{0}, \mathbf{w}_{2}\right) & =\frac{p\left(\mathbf{w}_{1} \mid \mathbf{w}_{0}\right) p\left(\mathbf{w}_{2} \mid \mathbf{w}_{1}, \mathbf{w}_{0}\right)}{\sum_{\mathbf{w}_{1}} p\left(\mathbf{w}_{1} \mid \mathbf{w}_{0}\right) p\left(\mathbf{w}_{2} \mid \mathbf{w}_{1}, \mathbf{w}_{0}\right)} \\
& =\frac{p\left(\mathbf{w}_{1} \mid \mathbf{w}_{0}\right) p\left(\mathbf{w}_{2} \mid \mathbf{w}_{1}\right)}{\sum_{\mathbf{w}_{1}} p\left(\mathbf{w}_{1} \mid \mathbf{w}_{0}\right) p\left(\mathbf{w}_{2} \mid \mathbf{w}_{1}\right)}
\end{aligned}
$$

thus

$$
\begin{aligned}
p\left(n_{1} \mid \mathbf{w}_{0}, \mathbf{w}_{2}\right)= & \frac{\sum_{\substack{\mathbf{w}_{1} \\
\text { s.t. } \operatorname{sum}\left(\mathbf{w}_{1}\right)=n_{1}}} p\left(\mathbf{w}_{1} \mid \mathbf{w}_{0}\right) p\left(\mathbf{w}_{2} \mid \mathbf{w}_{1}\right)}{\sum_{\mathbf{w}_{1}} p\left(\mathbf{w}_{1} \mid \mathbf{w}_{0}\right) p\left(\mathbf{w}_{2} \mid \mathbf{w}_{1}\right)} \\
= & \frac{\sum_{\substack{\mathbf{w}_{1} \\
\text { s.t. } \operatorname{sum}\left(\mathbf{w}_{1}\right)=n_{1}}} p\left(\mathbf{w}_{1} \mid \mathbf{w}_{0}\right) p\left(\mathbf{w}_{2} \mid \mathbf{w}_{1}\right)}{\sum_{n_{1}} \sum_{\substack{\mathbf{w}_{1} \\
\text { s.t. } \operatorname{sum}\left(\mathbf{w}_{1}\right)=n_{1}}} p\left(\mathbf{w}_{1} \mid \mathbf{w}_{0}\right) p\left(\mathbf{w}_{2} \mid \mathbf{w}_{1}\right)} .
\end{aligned}
$$

If the WITS are phenotypically identical, each bacterium has the same probability of surviving an antibiotic. Under this assumption, a distribution $\mathbf{w}_{1}$ of $n_{1}$ WITS in a bottleneck $\mathcal{P}_{1}$ can be regarded as a sample resulting from a random selection (without replacement) of $n_{1}$ WITS from the prebottleneck population $\mathcal{P}_{0}$ with distribution $\mathbf{w}_{0}$. The probability of selecting $\mathbf{w}_{1}$ from $\mathbf{w}_{0}$ without replacement such that $\operatorname{sum}\left(\mathbf{w}_{1}\right)=n_{1}$ is given by the multivariate hypergeometric probability distribution:

$$
p\left(\mathbf{w}_{1} \mid \mathbf{w}_{0}, \operatorname{sum}\left(\mathbf{w}_{1}\right)=n_{1}\right)=\frac{\left(\begin{array}{c}
w_{0}^{[1]} \\
w_{1}^{[1]}
\end{array}\right)\left(\begin{array}{c}
w_{0}^{[2]} \\
w_{1}^{[2]}
\end{array}\right) \cdots\left(\begin{array}{c}
w_{0}^{[8]} \\
w_{1}^{[8]}
\end{array}\right)}{\left(\begin{array}{l}
w_{0}^{[1]}+w_{0}^{[2]}+\cdots+w_{0}^{[8]} \\
w_{1}^{[1]}+w_{1}^{[2]}+\cdots+w_{1}^{[8]}
\end{array}\right)}
$$


As for $p\left(\mathbf{w}_{2} \mid \mathbf{w}_{1}\right)$, the independence between the WITS enables us to factorise $p\left(\mathbf{w}_{2} \mid \mathbf{w}_{1}, \boldsymbol{\theta}\right)$ as follows:

$$
p\left(\mathbf{w}_{2} \mid \mathbf{w}_{1}, \boldsymbol{\theta}\right)=\prod_{i} p\left(w_{2}^{[i]} \mid \mathbf{w}_{1}, \boldsymbol{\theta}\right)=\prod_{i} p\left(w_{2}^{[i]} \mid w_{1}^{[i]}, \boldsymbol{\theta}\right),
$$

and estimation of $p\left(w_{2}^{[i]} \mid w_{1}^{[i]}, \boldsymbol{\theta}\right)$ for (9) can be performed in the same manner as described for $p\left(n_{2} \mid n_{1}, \boldsymbol{\theta}\right)$ using Algorithm 1.

\subsection{On assuming proportionality}

Through Bayes' theorem, we have

$$
\begin{aligned}
p\left(n_{1} \mid \boldsymbol{\pi}_{0}, \mathbf{w}_{2}\right) & \propto p\left(n_{1} \mid \boldsymbol{\pi}_{0}\right) p\left(\mathbf{w}_{2} \mid n_{1}, \boldsymbol{\pi}_{0}\right) \\
& =p\left(n_{1}\right) p\left(\mathbf{w}_{2} \mid n_{1}, \boldsymbol{\pi}_{0}\right),
\end{aligned}
$$

where the elements of $\boldsymbol{\pi}_{0}$ are those of $\mathbf{w}_{0}$ expressed as relative frequencies.

Moreover, if we assume $p\left(n_{1}\right)$ to be equiprobable for all $n_{1}$ then

$$
p\left(n_{1} \mid \boldsymbol{\pi}_{0}, \mathbf{w}_{2}\right) \propto p\left(\mathbf{w}_{2} \mid n_{1}, \boldsymbol{\pi}_{0}\right) .
$$

Suppose we make the further assumption that the elements of $\mathbf{w}_{2}$ developed proportionaly from those in $\mathbf{w}_{1}: \mathbf{w}_{2}=\psi \mathbf{w}_{1}$ for some positive integer $\psi$. This assumption implies that $\psi n_{1}=\operatorname{sum}\left(\mathbf{w}_{2}\right)$ and, as $\operatorname{sum}\left(\mathbf{w}_{2}\right)$ is constant for a given $\mathbf{w}_{2}$, it follows that $\mathbf{w}_{2}$ can be derived from $\mathbf{w}_{1}$ using a range of $\psi$ values such that $\psi=\operatorname{sum}\left(\mathbf{w}_{2}\right) / n_{1}$. But is one $\psi$ more likely than another?

If $p\left(\mathbf{w}_{2} \mid n_{1}, \boldsymbol{\pi}_{0}\right)$ is defined by a multinomial distribution then

$$
\begin{aligned}
p\left(\mathbf{w}_{2} \mid n_{1}, \boldsymbol{\pi}_{0}\right) & =p\left(\left\langle w_{2}^{[1]}, w_{2}^{[2]}, \ldots, w_{2}^{[8]}\right\rangle \mid n_{1},\left\langle\pi_{0}^{[1]}, \pi_{0}^{[2]}, \ldots, \pi_{0}^{[8]}\right\rangle\right) \\
& =\left(\begin{array}{c}
w_{2}^{[1]}+w_{2}^{[2]}+\cdots+w_{2}^{[8]} \\
w_{2}^{[1]}, w_{2}^{[2]}, \ldots, w_{2}^{[8]}
\end{array}\right) \prod_{i=1}^{8}\left(\pi_{0}^{[i]}\right)^{[i]}
\end{aligned}
$$

however,

$$
\begin{aligned}
\left(\begin{array}{c}
\psi w_{1}^{[1]}+\psi w_{1}^{[2]}+\cdots+\psi w_{1}^{[8]} \\
\psi w_{1}^{[1]}, \psi w_{1}^{[2]}, \ldots, \psi w_{1}^{[8]}
\end{array}\right) \prod_{i=1}^{8}\left(\pi_{0}^{[i]}\right)^{\psi w_{1}^{[i]}}< & \\
& \left(\begin{array}{c}
w_{1}^{[1]}+w_{1}^{[2]}+\cdots+w_{1}^{[8]} \\
w_{1}^{[1]}, w_{1}^{[2]}, \ldots, w_{1}^{[8]}
\end{array}\right) \prod_{i=1}^{8}\left(\pi_{0}^{[i]}\right)^{[i]}
\end{aligned}
$$


for any positive integer $\psi$ (note that $\pi_{0}^{[i]}$ is the same on both sides of the inequality), thus

$$
p\left(\psi \mathbf{w}_{1} \mid n_{1}=\psi \operatorname{sum}\left(\mathbf{w}_{1}\right), \boldsymbol{\pi}_{0}\right)<p\left(\mathbf{w}_{1} \mid n_{1}=\operatorname{sum}\left(\mathbf{w}_{1}\right), \boldsymbol{\pi}_{0}\right) .
$$

Consequently, if $\mathbf{w}_{2}=\psi \mathbf{w}_{1}$ then $\operatorname{sum}\left(\mathbf{w}_{1}\right)$ is the most probable value for $n_{1}$. Put another way, if proportionality is assumed then the most probable value for $n_{1}$ is $\operatorname{sum}\left(\mathbf{w}_{2}\right)$ divided by the highest common factor for the elements of $\mathbf{w}_{2}$.

\subsection{A sampling approach}

In the previous section, we have shown how to obtain values for $p\left(\mathbf{w}_{1} \mid \mathbf{w}_{0}\right)$ and $p\left(\mathbf{w}_{2} \mid \mathbf{w}_{1}\right)$ that are required for (7), but (7) also requires us to determine the summands for every possible $\mathbf{w}_{1}$ such that $\sum_{i} w_{1}^{[i]}=n_{1}$. The problem with this is that the number of possible $\mathbf{w}_{1}$ for a given value of $n_{1}$ grows super-exponentially with $n_{1}$ (Charalambides, 2002, p.138); for example, the number of possible $\mathbf{w}_{1}$ when selecting 100 microbes from $\mathbf{w}_{0}=$ $\left\langle 1000^{[1]}, 1000^{[2]}, \ldots, 1000^{[8]}\right\rangle$ is more than 26 thousand million. As this is combinatorially (and thus computationally) challenging, an alternative approach is required.

To circumvent the combinatorial issue, one could consider restricting the summations of (7) to the more probable configurations of $\mathbf{w}_{1}$, such as the modes of $\mathbf{w}_{1}$. An algorithm for the generation of all the modes of a multivariate hypergeometric distribution has been proposed by Requena and Cludad (2003), but a simpler approach is to randomly sample points $\mathbf{w}_{1}$, say 1000 times, from $p\left(\mathbf{w}_{1} \mid \mathbf{w}_{0}\right)$ as a multivariate hypergeometric distribution, given that most of these points would be expected to be in the vicinity of the modes.

Our implementation of the sampling approach is shown in Algorithm 2, and its efficacy was tested using the following steps of a toy experiment:

1: Set $\mathbf{w}_{0}$ to $\left\langle 600^{[1]}, 600^{[2]}, \ldots, 600^{[8]}\right\rangle$

2: Choose a target bottleneck size $n_{1}^{\star} \in\{80,800,1600\}$

3: In order to choose a $\mathbf{w}_{1}$ associated with target $n_{1}^{\star}$, select a mode $\mathbf{w}_{1}^{\star}$ from the multivariate hypergeometric distribution associated with random samples of size $n_{1}^{\star}$ taken from $\mathbf{w}_{0}$ (Requena \& Cludad, 2003)

4: In order to choose $\mathbf{a} \mathbf{w}_{2}$ resulting from $\mathbf{w}_{2}$, first do 


$$
\left\{\left(\mathbf{w}_{2}\right)_{j}\right\}_{j=1, \ldots, 101} \stackrel{i . i . d}{\sim} \operatorname{Gillespie}\left(\mathbf{w}_{1}^{\star}, \Delta t, \boldsymbol{\theta}\right)
$$

5: then, for $\mathbf{w}_{2}$, set $\mathbf{w}_{2}^{\star}$ to the median of $\left\{\left(\mathbf{w}_{2}\right)_{j}\right\}_{j=1, \ldots, 101}$

6: Obtain $\widehat{p}\left(n_{1} \mid \mathbf{w}_{0}, \mathbf{w}_{2}^{\star}\right)$ using Algorithm 2

7: Compare $\widehat{p}\left(n_{1} \mid \mathbf{w}_{0}, \mathbf{w}_{2}^{\star}\right)$ with target $n_{1}^{\star}$

Figure 6 displays the resulting posterior distributions, which have median accuracies similar to those shown for the estimation of $p\left(n_{1} \mid n_{2}\right)$ derived by Algorithm 1.

\section{Patterns of missing WITS}

An assumption made when estimating $p\left(n_{2} \mid n_{1}, \boldsymbol{\theta}\right)$ via Gillespie simulation is that parameters $\boldsymbol{\theta}$ are known and are not influenced by the presence of an antibiotic, but this is not necessarily always the case (Kaiser et al., 2014). Consequently, how can we estimate bottleneck size when $\boldsymbol{\theta}$ is not known to us?

Consider (7) written as

$$
p\left(n_{1} \mid \mathbf{w}_{0}, \mathbf{w}_{2}\right) \propto \sum_{\substack{\mathbf{w}_{1} \\ \text { s.t. } \operatorname{sum}\left(\mathbf{w}_{1}\right)=n_{1}}} p\left(\mathbf{w}_{1} \mid \mathbf{w}_{0}\right) p\left(\mathbf{w}_{2} \mid \mathbf{w}_{1}\right)
$$

and suppose we replace $\mathbf{w}_{2}$ with a vector $\xi_{2}$ denoting which WITS in $\mathbf{w}_{2}$ are missing, then $p\left(\mathbf{w}_{2} \mid \mathbf{w}_{1}\right)$, in turn, becomes replaced by $p\left(\xi_{2} \mid \mathbf{w}_{1}\right)$. Furthermore, if we assume that missingness pattern $\xi_{2}$ is equal to the missingness pattern $\xi_{1}$ of $\mathbf{w}_{1}$ then $\xi_{2}$ is implied by $\mathbf{w}_{1}$ and there is no need to consider postbottleneck dynamics. Using this approach, (11) simplifies to

$$
p\left(n_{1} \mid \mathbf{w}_{0}, \xi_{2}\right) \propto \sum_{\substack{\mathbf{w}_{1} \\ \text { s.t. } \operatorname{sum}\left(\mathbf{w}_{1}\right)=n_{1}}} p\left(\mathbf{w}_{1} \mid \mathbf{w}_{0}\right) \mathbb{1}\left(\mathbf{w}_{1} \Rightarrow \xi_{2}\right),
$$

where $\mathbb{1}(\cdot)$ is the indicator function. However, the assumption that $\xi_{2}=\xi_{1}$, and thus that $\mathbf{w}_{1} \Rightarrow \xi_{2}$, may not hold if a few WITS are randomly lost soon after a bottleneck due to a combination of (a) a small bottleneck, (b) a large number of WITS, and (c) high post-bottleneck replication and death rates.

In order to compare the accuracy of (11) with (12), an experiment was used based on the following scenario. A bottleneck WITS population $\mathbf{w}_{1}$ of size $n_{1}$ is assumed to have been sampled from $\mathbf{w}_{1}=\left\langle 4^{[1]}, 4^{[2]}, \ldots, 4^{[8]}\right\rangle$ without 
replacement. Each element $w_{1}^{[i]}$ of $\mathbf{w}_{1}$ then gives rise to an element $w_{2}^{[i]}$ of $\mathbf{w}_{2}$ by sampling from a Poisson distribution with Poisson parameter $\lambda=10 w_{1}^{[i]}$. This scenario is the basis for the following toy experiment:

1: Set $\mathbf{w}_{0}$ to $\left\langle 4^{[1]}, 4^{[2]}, \ldots, 4^{[8]}\right\rangle$

2: Choose a target bottleneck size $n_{1}^{\star} \in\{6,7,8,20\}$

3: In order to choose a $\mathbf{w}_{1}$ associated with target $n_{1}^{\star}$, select a mode $\mathbf{w}_{1}^{\star}$ from the multivariate hypergeometric distribution associated with random samples of size $n_{1}^{\star}$ taken from $\mathbf{w}_{0}$

4: For $\mathbf{w}_{2}$, use $\mathbf{w}_{2}^{\star}=10 \mathbf{w}_{1}^{\star}$ (i.e., vector of expected values from the Poisson distributions)

5: Get missingness pattern $\xi_{2}$ corresponding to $\mathbf{w}_{2}^{\star}$

6: Obtain $p\left(n_{1} \mid \mathbf{w}_{0}, \mathbf{w}_{2}^{\star}\right)$ using (11)

7: Compare $p\left(n_{1} \mid \mathbf{w}_{0}, \mathbf{w}_{2}^{\star}\right)$ with target $n_{1}^{\star}$

8: Obtain $p\left(n_{1} \mid \mathbf{w}_{0}, \xi_{2}\right)$ using (12)

9: Compare $p\left(n_{1} \mid \mathbf{w}_{0}, \xi_{2}\right)$ with target $n_{1}^{\star}$

The results of the experiment are shown in Figures 8 and 9. In the case of the probability mass functions for $p\left(n_{1} \mid \mathbf{w}_{0}, \mathbf{w}_{2}^{\star}\right)$, the modes coincided exactly with the target values, but this was not the case for $p\left(n_{1} \mid \mathbf{w}_{0}, \xi_{2}\right)$. When at least one WITS was missing, the mode for $p\left(n_{1} \mid \mathbf{w}_{0}, \xi_{2}\right)$ was greater than the target value. This is associated with the observation that the mean value of $p\left(\mathbf{w}_{2} \mid \mathbf{w}_{1}\right)$ as encountered in (11) tended to be less than the mean value for $\mathbb{1}\left(\mathbf{w}_{1} \Rightarrow \xi_{2}\right)$ in $(12)$, which is equal to $p\left(\mathbf{w}_{1} \Rightarrow \xi_{2}\right)$. When no WITS were missing, the resulting probability mass function for $p\left(n_{1} \mid \mathbf{w}_{0}, \xi_{2}\right)$ exhibited a plateau as $n_{1}$ increased. This can be explained as follows: it is increasingly unlikely that no WITS are missing as $n_{1}$ decreases; on the other hand, the absence of missing WITS can be explained by the occurrence of large $n_{1}$ values up to and including the complete absence of a bottleneck.

When $p\left(n_{1} \mid \mathbf{w}_{0}, \mathbf{w}_{2}\right)$ has a plateau, a lower bound for $n_{1}$ can be set equal to the lower bound of the $95 \%$ highest density interval with respect to $p\left(n_{1} \mid \mathbf{w}_{0}, \mathbf{w}_{2}\right)$, which is a type of $95 \%$ credible interval.

Note that the above toy experiment uses Equation (12) exactly so as to display the resulting distributions. In reality, the inoculum size would be far greater than $4 \times 8$ and, in such circumstances, the sampling approach of Section 3.3 would be used instead. Figure shows the result of using sampling when $\xi_{2}$ is used in place of $\mathbf{w}_{2}$, with $n_{1}=600 \times 8$. 


\subsection{Dilution of WITS with monotypes}

In Figure 9 (c), the bottleneck of size 20 could not be estimated because of the presence of a plateau instead of a mode. This issue can be overcome by diluting the WITS with untagged (i.e., monotype) isogenic bacteria. The justification for this is that, for a fixed $n_{1}$, the probability of at least one WITS being missing increases as the pre-bottleneck population $\mathbf{w}_{0}$ becomes more dilute.

Let $\mathbf{w}_{0}^{+}$represent $\mathbf{w}_{0}$ augmented with $u$ untagged bacteria. For example, if we add 10 untagged bacteria to $\mathbf{w}_{0}=\left\langle 4^{[1]}, \ldots, 4^{[8]}\right\rangle$ then $\mathbf{w}_{0}^{+}=$ $\left\langle 4^{[1]}, \ldots, 4^{[8]}, 10\right\rangle$. Upon using $\mathbf{w}_{0}^{+}$in place of $\mathbf{w}_{0}$, expression $(12)$ becomes

$$
p\left(n_{1} \mid \mathbf{w}_{0}^{+}, \xi_{2}\right) \propto \sum_{\substack{\mathbf{w}_{1}^{+} \\ \text {s.t. } \operatorname{sum}\left(\mathbf{w}_{1}^{+}\right)=n_{1}}} p\left(\mathbf{w}_{1}^{+} \mid \mathbf{w}_{0}^{+}\right) \mathbb{1}\left(\mathbf{w}_{1}^{+} \Rightarrow \xi_{2}\right),
$$

where $\mathbf{w}_{1}^{+}$allows for the possibility that untagged bacteria can be present in the bottleneck. Implication $\mathbf{w}_{1}^{+} \Rightarrow \xi_{2}$ in (13) is based only on the WITS component of $\mathbf{w}_{1}^{+}$; the untagged bacteria in $\mathbf{w}_{1}^{+}$are ignored.

By way of example, suppose that we add $u=13$ untagged bacteria to $\mathbf{w}_{0}=\left\langle 4^{[1]}, \ldots, 4^{[8]}\right\rangle$ in order to perform the following toy experiment:

1: Set $\mathbf{w}_{0}^{+}$to $\left\langle 4^{[1]}, 4^{[2]}, \ldots, 4^{[8]}, u\right\rangle$

2: Set number of untagged bacteria $u=13$

3: Set target bottleneck size $n_{1}^{\star}=20$

4: In order to choose a $\mathbf{w}_{1}^{+}$associated with target $n_{1}^{\star}$, first select a mode $\mathbf{w}_{1}^{\star}$ from the multivariate hypergeometric distribution associated with random samples of size $n_{1}^{\star}-u$ taken from $\left\langle 4^{[1]}, 4^{[2]}, \ldots, 4^{[8]}\right\rangle$, and then set $\mathbf{w}_{1}^{+\star}=\left\langle\mathbf{w}_{1}^{\star}, u\right\rangle$

5: For $\mathbf{w}_{2}$, use $\mathbf{w}_{2}^{\star}=10 \mathbf{w}_{1}^{+\star}$

6: Get missingness pattern $\xi_{2}$ corresponding to $\mathbf{w}_{2}^{\star}$

7: Obtain $p\left(n_{1} \mid \mathbf{w}_{0}^{+}, \xi_{2}\right)$ using (13)

Figure 10 shows that dilution with untagged bacteria has allowed an estimate of the bottleneck size to be performed. But a note of caution is due. Using $u=13$ allowed the bottleneck size to be estimated as 18 (whereas $u=12$ gave a plateau), but the position of the mode for $p\left(n_{1} \mid \mathbf{w}_{0}^{+}, \xi_{2}\right)$ is influenced by the choice of $u$, with the mode decreasing as $u$ increases. For example, the mode was 13 when $u=14$ and 10 when $u=15$. A similar behaviour has 
been observed when using other target values for $n_{1}$. This suggests that one should use the smallest possible value for $u$ that permits a mode to appear instead of a plateau.

The above WITS dilution technique was used by Maier et al. (2014) to estimate the size of gut luminal bottlenecks during Salmonella Typhimurium colitis. The inoculum consisted of seven WITS in equal proportions, which was increasingly diluted with an untagged isogenic wild-type strain until a loss of at least one WITS was first detected in a post-bottleneck population. This point occurred at a dilution of 1:7000. The size of a bottleneck was then estimated using a likelihood function based on binomial selection.

Lim et al. (2014) also developed a method to estimate the size of a bottleneck from an observation of missing WITS, but their approach was more granular in that it was restricted to just those cases where at least one WITS was missing but without considering the precise the number of missing WITS. Let $\zeta \in\{$ true, false $\}$ denote the state that at least one WITS is missing from $\mathbf{w}_{2}$ (and thus assumably from $\mathbf{w}_{1}$ ). In this context, the posterior for bottleneck size is $p\left(n_{1} \mid \mathbf{w}_{0}, \zeta\right)$. The computational approach used by Lim et al. differed from (12) in that they derived a plot of $\widehat{p}\left(\zeta \mid n_{1}, \mathbf{w}_{0}\right)$ as a function of $n_{1}$ using computer simulations and then assumed that

$$
p\left(n_{1} \mid \mathbf{w}_{0}, \zeta\right) \propto p\left(\zeta \mid n_{1}, \mathbf{w}_{0}\right)
$$

\subsection{On increasing the number of WITS}

All the examples shown so far have been based on the use of eight WITS, but what if a larger number of WITS are used? Intuitively, if we keep the bottleneck size $n_{1}$ constant but increase the number $|\mathcal{W}|$ of WITS available then the number of missing WITS is expected to increase. Furthermore, the rate of change in the number of missing WITS as $n_{1}$ decreases is expected to increase as $|\mathcal{W}|$ increases. This suggests that accuracy in the estimation of $n_{1}$ from missingness patterns $\xi_{2}$ should improve with larger $|\mathcal{W}|$. This argument is supported by the results of the computer simulations conducted by Lim et al. (2014) using different values for $|\mathcal{W}|$ in which a significant improvement occurs on going from $|\mathcal{W}|=10$ to $|\mathcal{W}|=40$.

As a further demonstration, the posterior distribution $p\left(n_{1} \mid \mathbf{w}_{0}, \xi_{2}\right)$ shown in Figure 9 when $n_{1}=7$, which is based on 8 WITS, was recalculated using 12 WITS resulting in a decrease in variance (Figure 11). 


\section{Discussion}

We describe Bayesian approaches to estimating the size of bacterial bottlenecks given observation of a post-bottleneck population (either monotype or multitype), but size estimation with respect to monotypes is possible only when post-bottleneck dynamics is known (or assumed).

The use of multitype isogenic bacteria in the form of WITS allow the composition of pre-bottleneck populations to be included in the analysis. Furthermore, the use of WITS enables bottleneck sizes to be estimate when post-bottleneck dynamics is not known. This is done through observation of patterns of missing WITS; however, this can require dilution of an inoculum with isogenic untagged bacteria. Figure 12 provides a flowchart that summarises these observations.

\section{Author contributions}

$\mathrm{RD}, \mathrm{PM}$ and $\mathrm{OR}$ conceived the project. RD performed the mathematical analyses and simulations, which were checked by DJP. All the authors contributed to the preparation of the manuscript.

\section{Conflict of interest}

The authors declare that they have no conflicts of interest.

\section{Acknowledgements}

RD was supported by BBSRC grant BB/I002189/1 awarded to PM. DJP was supported by BBSRC grant BB/M020193/1 awarded to OR.

\section{References}

Abel, S., Abel zur Wiesch, P., Davis, B., \& Waldor, M. (2015). Analysis of bottlenecks in experimental models of infection. PLoS Pathogens, $11(6)$, e1004823.

Aerts, M., Augustyns, I., \& Janssen, P. (1997a). Smoothing sparse multinomial data using local polynomial fitting. Journal of Nonparametric Statistics, 8(2), 127-147. 
Aerts, M., Augustyns, I., \& Janssen, P. (1997b). Sparse consistency and smoothing for multinomial data. Statistics and Probability Letters, 33, 41-48.

Charalambides, C. (2002). Enumerative combinatorics. Boca Raton, Fl: Chapman \& Hall/CRC.

Coward, C., Restif, O., Dybowski, R., Grant, A., Maskell, D., \& Mastroeni, P. (2014). The effects of vaccination and immunity on bacterial infection dynamics in vivo. PLoS Pathogens, $10(9)$, e1004359.

Crimmins, G., \& Isberg, R. (2012). Analyzing microbial disease at high resolution: following the fate of the bacterium during infection. Current Opinion in Microbiology, 15(1), 23-27.

Dougan, G., \& Baker, S. (2014). Salmonella enterica serovar Typhi and the pathogenesis of typhoid fever. Annual Review of Microbiology, 68, 317-336.

Dybowski, R., Restif, O., Goupy, A., Maskell, D., Mastroeni, P., \& Grant, A. (2015). Single passage in mouse organs enhances the survival and spread of Salmonella enterica. Journal of the Royal Society Interface, 12, 20150702.

Gerlini, A., Colomba, L., Furi, L., Braccini, T., Manso, A. S., Pammolli, A., et al. (2014). The role of host and microbial factors in the pathogenesis of pneumococcal bacteraemia arising from a single bacterial cell bottleneck. PLoS Pathogens, $10(3)$, e1004026.

Gillespie, D. (1997). Exact stochastic simulation of coupled chemical reactions. Journal of Physical Chemistry, 81, 2340-236.

Grant, A., Restif, O., McKinley, T., Sheppard, M., Maskell, D., \& Mastroeni, P. (2008). Modelling within-host spatiotemporal dynamics of invasive bacterial disease. PLoS Biology, 6(4), e74.

Jacob, P., \& Oliveira, P. (2011). Relative smoothing of discrete distributions with sparse observations. Journal of Statistical Computation and Simulation, 81(1), 109-121.

Kaiser, P., Regoes, R., Dolowschiak, T., Wotzka, S., Lengefeld, J., Slack, E., et al. (2014). Cecum lymph node dendritic cells harbor slow-growing bacteria phenotypically tolerant to antibiotic treatment. PLoS Biology, 12(2), e1001793.

Kono, M., Zafar, M., Zuniga, M., Roche, A., Hamaguchi, S., \& Weiser, J. (2016). Single cell bottlenecks in the pathogenesis of Streptococcus pneumoniae. PLoS Pathogens, 12(10), e1005887.

Krimbas, C., \& Tsakas, S. (1971). The genetics of Dacus okae. V. Changes 
of esterase polymorphism in a natural populatian following insecticide control-selection or drift? Evolution, 25, 454-460.

Lim, C., Voedisch, S., Wahl, B., Rouf, S., Geffers, R., Rhen, M., et al. (2014). Independent bottlenecks characterize colonization of systemic compartments and gut lymphoid tissue by Salmonella. PLoS Pathogens, 10(7), e1004270.

Lowe, D., Ernst, S., Zito, C., Ya, J., \& Glomski, I. (2013). Bacillus anthracis has two independent bottlenecks that are dependent on the portal of entry in an intranasal model of inhalational infection. Infection $\mathscr{G} \mathrm{Im}$ munity, 81 (12), 4408-4420.

Maier, L., Diard, M., Sellin, M., Chouffane, E.-S., Trautwein-Weidner, K., Periaswamy, B., et al. (2014). Granulocytes impose a tight bottleneck upon the gut luminal pathogen population during Salmonella Typhimurium colitis. PLoS Pathogens, 10(12), e1004557.

Pamilo, P., \& Varvio-Aho, S. (1980). On the estimation of population size from allele frequency changes [Letter to the Editor]. Genetics, 95(4), 1055-1057.

Requena, F., \& Cludad, M. (2003). The maximum probability 2 x c contingency tables and the maximum probability points of the multivariate hypergeometric distribution. Communications in Statistics - Theory and Methods, 32(9), 1737-1752.

Ruppert, D., \& Wand, M. (1994). Multivariate locally weighted least squares regression. Annals of Statistics, 22, 1346-1370.

Schwartz, D., Chen, S., Hultgren, S., \& Seed, P. (2011). Population dynamics and niche distribution of uropathogenic Escherichia coli during acute and chronic urinary tract infection. Infection \& Immunity, 79(10), 4250-4259.

Simonoff, J. (1996). Smoothing methods in statistics. New York: SpringerVerlag.

Sourdis, J., \& Krimbas, C. (1980). On Pamilo and Varvio-Aho's note about the estimation of effective population size [Letter to the Editor]. Genetics, 96(2), 561-563. 


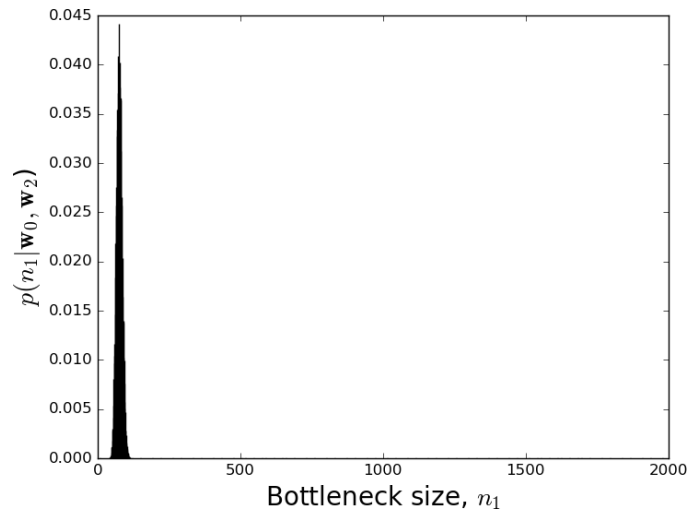

(a)

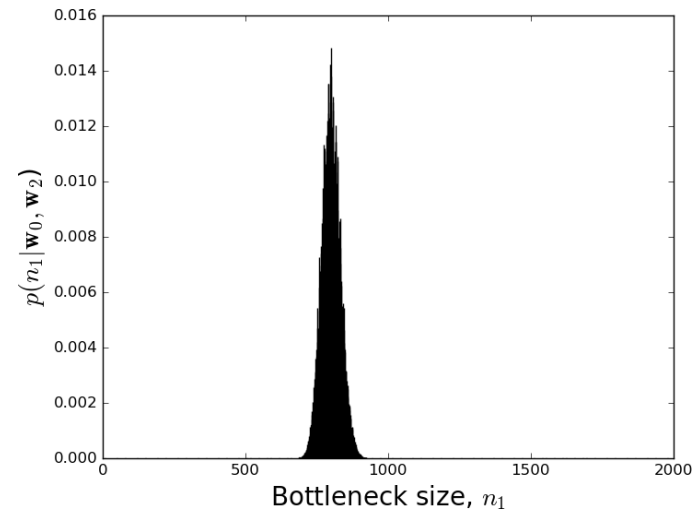

(b)

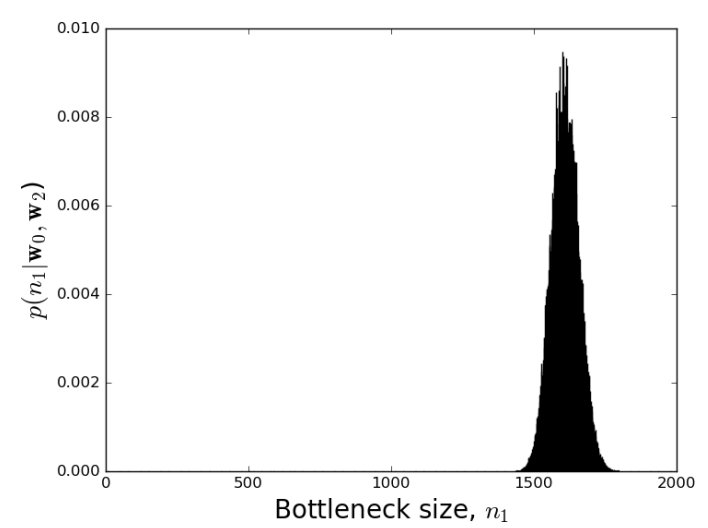

(c)

Figure 6: Posterior bottleneck-size distributions $p\left(n_{1} \mid \mathbf{w}_{0}, \mathbf{w}_{2}\right)$ estimated using Algorithm 2. Target bottlenecks of size (a) $n_{1}=80$, (b) $n_{1}=800$, and (c) $n_{1}=1600$, were artificially induced. See Section 3.3 for details. 


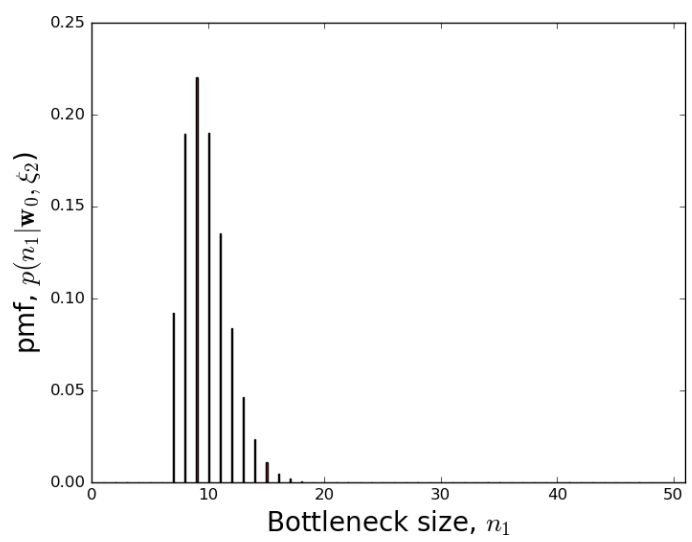

(a)

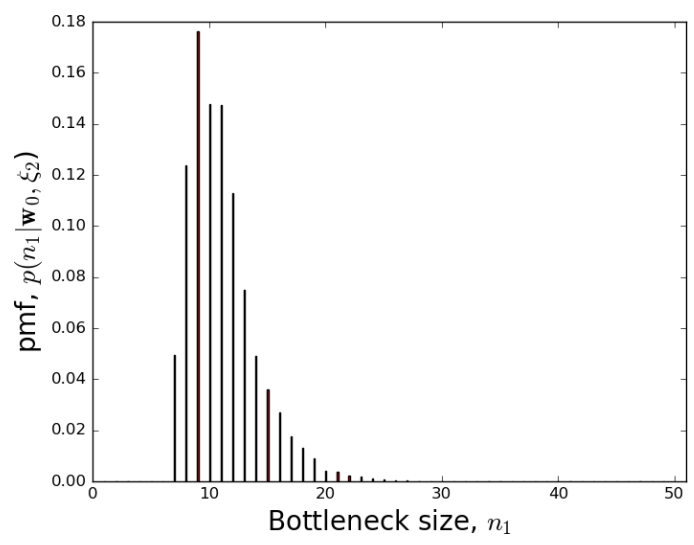

(b)

Figure 7: Posterior bottleneck-size distributions obtained (a) exactly using equation (12) with $\mathbf{w}_{0}=$ $\left\langle 4^{[1]}, 4^{[2]}, \ldots, 4^{[8]}\right\rangle$, and (b) estimated using 1000 samples with $\mathbf{w}_{0}=\left\langle 600^{[1]}, 600^{[2]}, \ldots, 600^{[8]}\right\rangle$. Target is $n_{1}=7$ in both cases. 


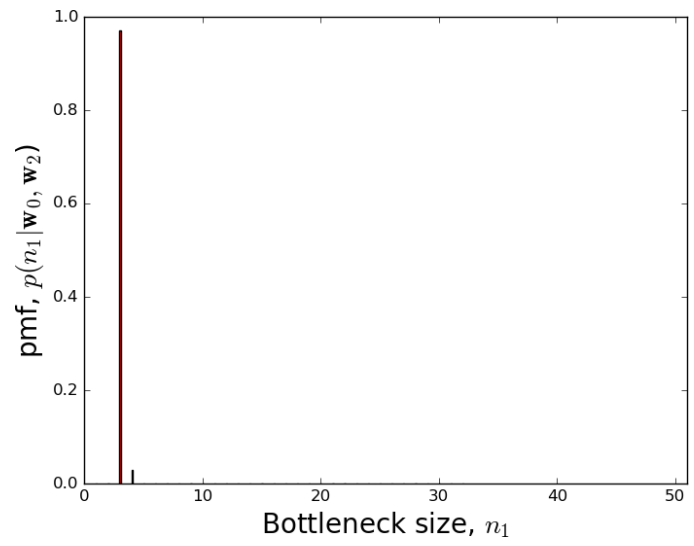

(a)

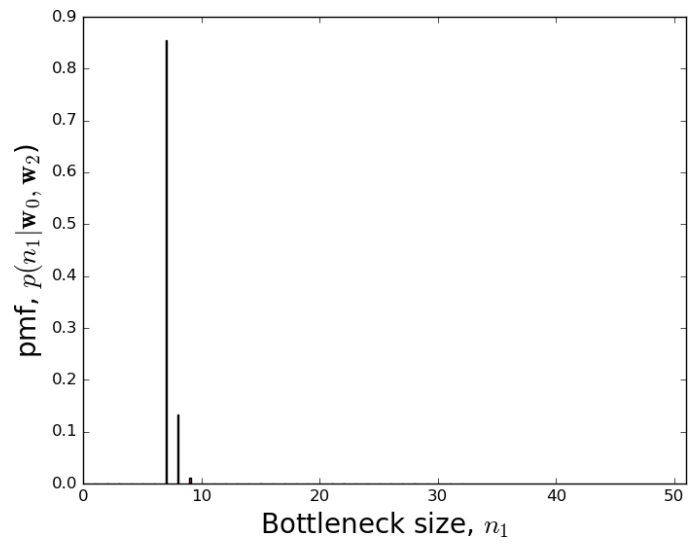

(b)

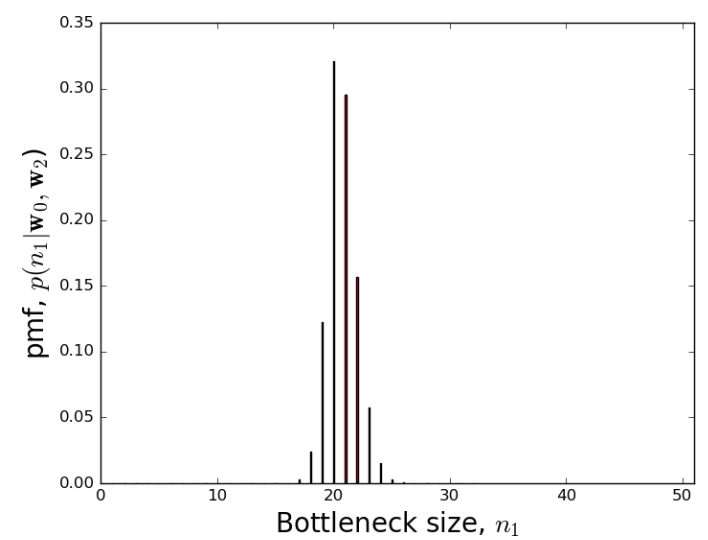

(c)

Figure 8: Posterior bottleneck-size distributions $p\left(n_{1} \mid \mathbf{w}_{0}, \mathbf{w}_{2}\right)$ determined accurately using (11). Target bottlenecks of size (a) $n_{1}=3$, (b) $n_{1}=7$, and (c) $n_{1}=20$, were used. See Section 4 for details. 


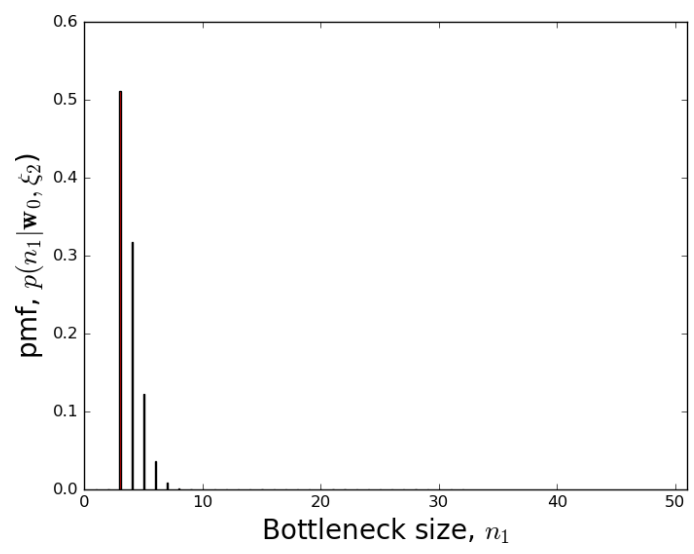

(a)

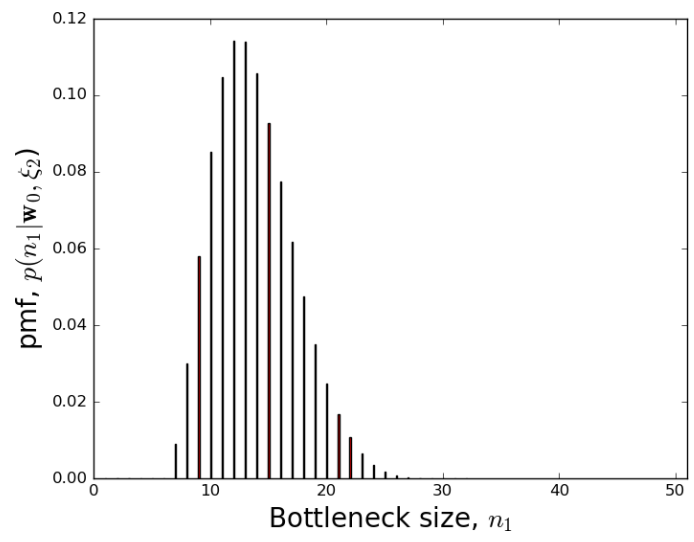

(b)

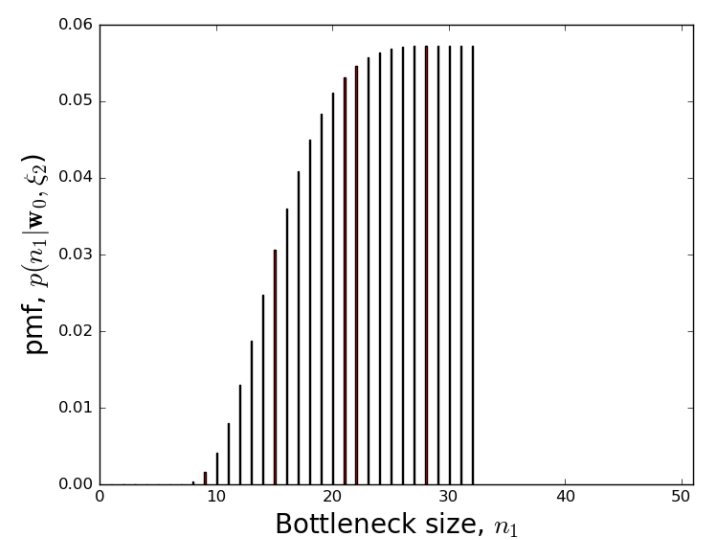

(c)

Figure 9: Posterior bottleneck-size distributions $p\left(n_{1} \mid \mathbf{w}_{0}, \xi_{2}\right)$ determined accurately using (12). Target bottlenecks of size (a) $n_{1}=3$, (b) $n_{1}=7$, and (c) $n_{1}=20$, were used. See Section 4 for details. 


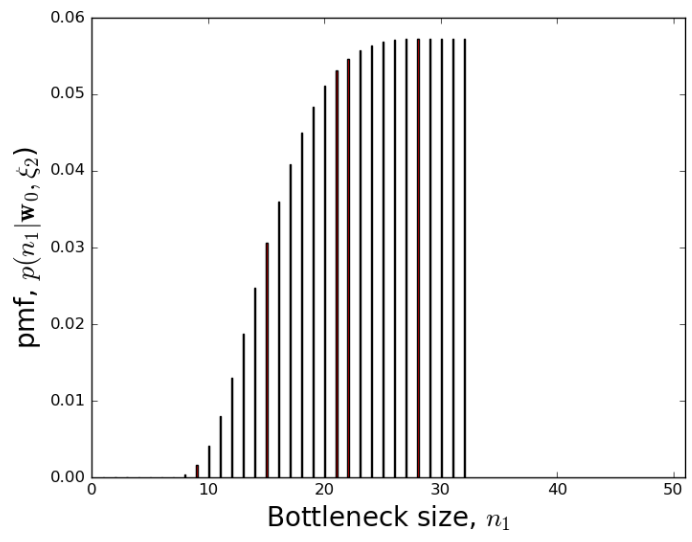

(a)

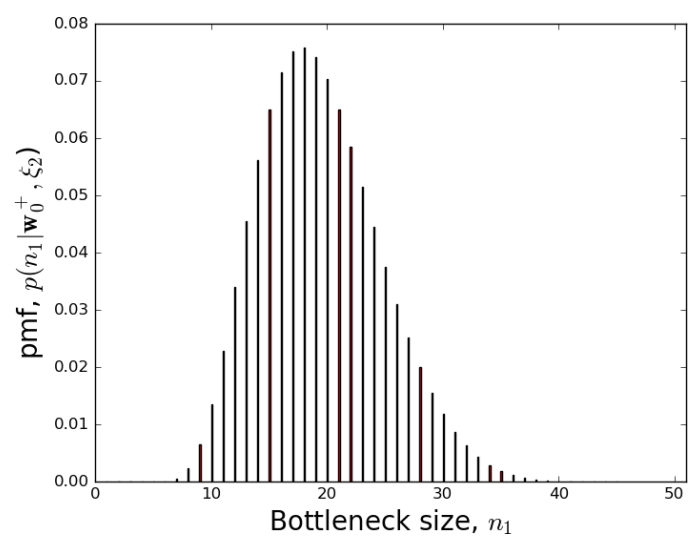

(b)

Figure 10: Posterior bottleneck-size distributions (a) without dilution and (b) with dilution. Distributions $p\left(n_{1} \mid \mathbf{w}_{0}, \xi_{2}\right)$ and $p\left(n_{1} \mid \mathbf{w}_{0}^{+}, \xi_{2}\right)$ were determined accurately using (12) and (13), respectively. The target bottleneck size was $n_{1}=20$ in both cases. For the dilution, $u=13$ untagged bacteria were present in $\mathbf{w}_{0}^{+}$. See Section 4.1 for details. 


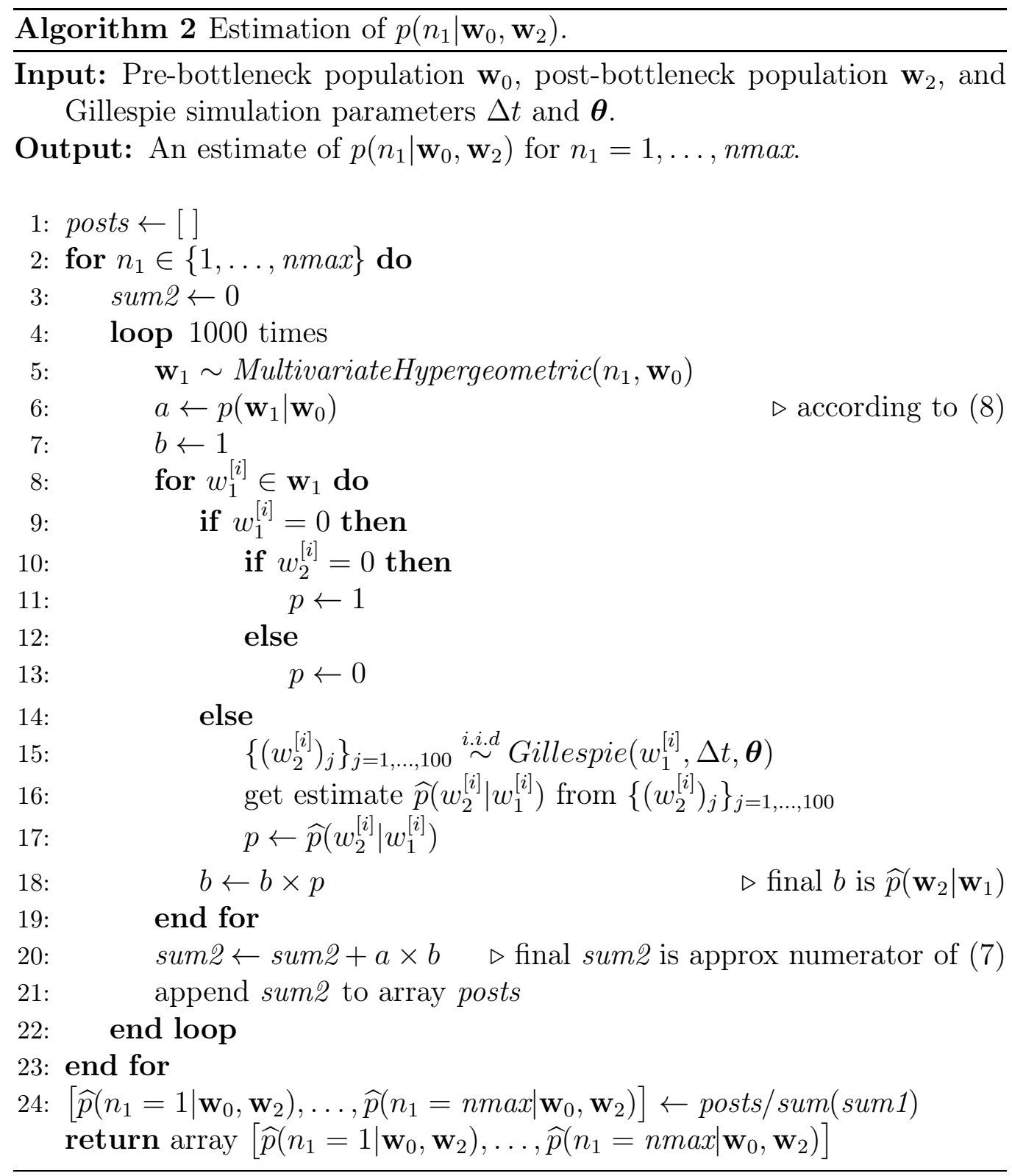




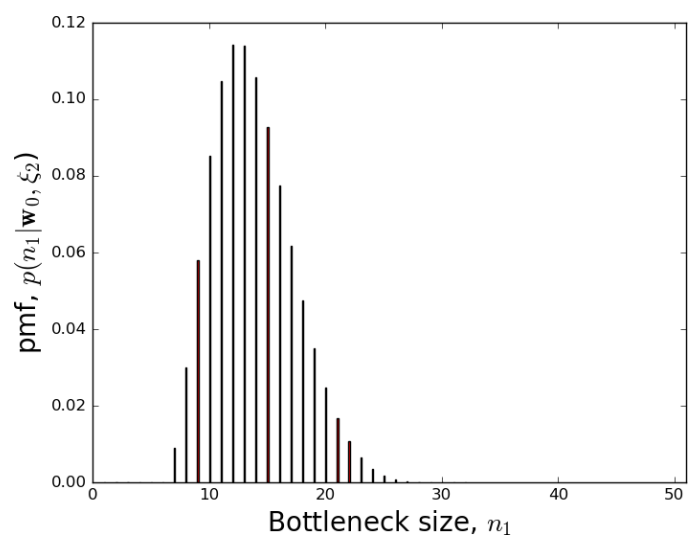

(a)

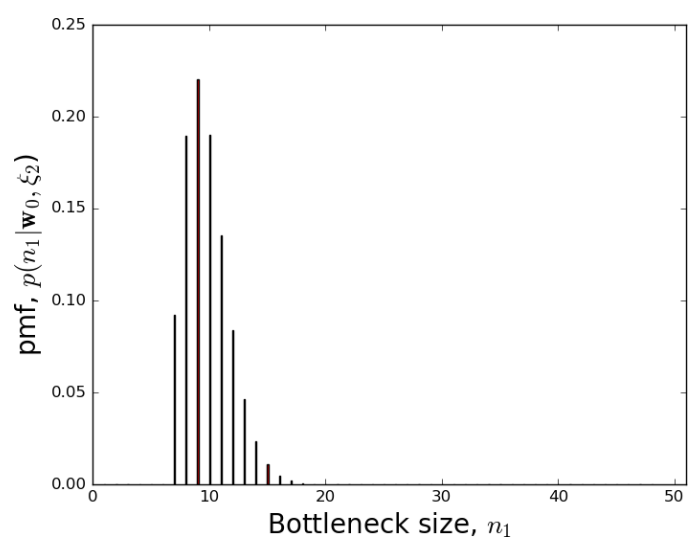

(b)

Figure 11: Posterior bottleneck-size distributions obtained (a) with 8 WITS and (b) with 12 WITS. Note the decrease in variance. 


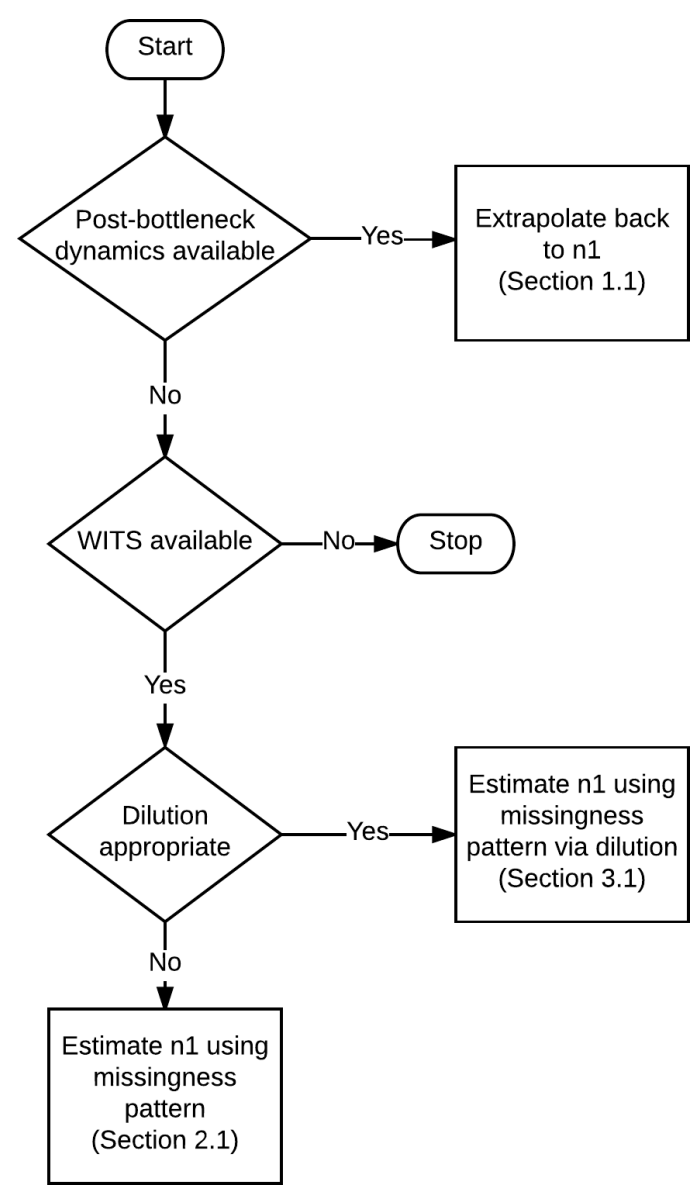

Figure 12: Flowchart summarizing alternative strategies to bottleneck-size estimation depending on circumstances. 